\title{
ON SOME QUESTIONS OF UNIQUENESS IN THE THEORY OF SYMMETRIZATION
}

\author{
MATTS ESSÉN and DANIEL F. SHEA*
}

Introduction. In a series of remarkable papers, [1]-[4], A. Baernstein has solved a collection of extremal problems in function theory, potential theory and harmonic analysis. A central theme in these papers is the relation between plane domains $D$ and their symmetrizations $D^{*}$, and majorization arguments showing the extremality of certain rearrangements of functions associated with the domains $D$.

For example, in [2] Baernstein studies the class $S$ of schlicht functions in the unit disk $U=\{|z|<1\}$, normalized by $f(0)=0, f^{\prime}(0)=1$. He proves

$$
\int_{0}^{2 \pi} \Phi\left(\log \left|f\left(r e^{i \theta}\right)\right|\right) d \theta \leqq \int_{0}^{2 \pi} \Phi\left(\log \left|k\left(r e^{i \theta}\right)\right|\right) d \theta \quad(0<r<1)
$$

for any $\Phi$ convex and increasing on $(-\infty, \infty)$ and for all $f \in S$, with $k(z)=z /(1-z)^{2}$. Further, Baernstein proves that if equality holds in (1) for some increasing, strictly convex $\Phi$ and some $r \in(0,1)$, then

$$
f(z)=e^{-i \alpha} k\left(e^{i \alpha} z\right)
$$

for some real $\alpha$.

(A convex function is termed strictly convex if it is not linear on any interval.)

Baernstein finds other inequalities analogous to (1) for general analytic functions in $U$, Green's functions, harmonic measures and conjugate functions; but for these results he leaves open the question of finding uniqueness results like (2) for the expected extremal functions.

Our purpose here is to consider this question, and we first study the case of equality in Baernstein's result

$$
\int_{0}^{2 \pi} \Phi\left(u\left(r e^{i \theta}\right)\right) d \theta \leqq \int_{0}^{2 \pi} \Phi\left(v\left(r e^{i \theta}\right)\right) d \theta \quad(0<r<\infty)
$$

\footnotetext{
* Research supported in part by NSF grant GP-21340.
} 
for Green's functions: Let $D$ be a domain in the extended plane having a Green's function, let $z_{0} \in D$ and let $u$ be Green's function of $D$ with pole at $z_{0}$. Let $D^{*}$ be the circular symmetrization, with respect to the ray $\theta=0$, of $D$ (the explicit definition is given in Section 1), and let $v$ be Green's function of $D^{*}$ with pole at $\left|z_{0}\right|$. Define $u$ (respectively $v$ ) to be $\equiv 0$ outside of $D$ (resp. $D^{*}$ ). Then Baernstein has proved (3) for all convex nondecreasing functions $\Phi$ on $[0, \infty)$; see [2], Theorem 5.

To this we add

Theorem 1. If equality holds in (3) for some $r \in D^{*} \cap(0, \infty)$ and some increasing strictly convex $\Phi$, then

$$
D=e^{i \alpha} D^{*}
$$

for some real $\alpha$. If $z_{0} \neq 0$ or $\infty$, then $\alpha=\arg z_{0}$.

In case the $r$-value for which equality is known to hold is $r=\left|z_{0}\right|, \cdot$ it is to be understood here that $\Phi$ satisfies also $\int_{0}^{1} \Phi(-\log \theta) d \theta<\infty$, so that the $\Phi$-means of $u$ and $v$ on this circle remain finite.

Theorem 1 answers a question posed in [2], p. 142.

Put $M(r, u)=\sup _{\theta} u\left(r e^{i \theta}\right)$ and notice that (3) implies

$$
M(r, u) \leqq M(r, v) \quad(0<r<\infty) .
$$

Theorem 1 suggests that equality here for some $r \in D^{*} \cap(0, \infty), r \neq\left|z_{0}\right|$, implies (4), and we prove this as well in Section 2.

An analogue of Theorem 1 for integral means of harmonic measures is given in Section 2.

Inequality (3) is a key element in the proof of a striking symmetrization principle for functions holomorphic in a disk established in [2]: Let $f$ be holomorphic in $U$, put $D=f(U)$ and let $D_{0}$ be a simply connected domain, $D_{0} \supset D^{*}$. Assume $D_{0}$ is not the whole plane, so that there exists a conformal map $F$ of $U$ onto $D_{0}$ with $F(0)=|f(0)|$. Then Baernstein proved

$$
\int_{0}^{2 \pi} \Phi\left(\log \left|f\left(r e^{i \theta}\right)\right|\right) d \theta \leqq \int_{0}^{2 \pi} \Phi\left(\log \left|F\left(r e^{i \theta}\right)\right|\right) d \theta \quad(0<r<1)
$$

for all convex nondecreasing $\Phi$ on $(-\infty, \infty)$.

Using Theorem 1, we prove

Theorem 2. If equality holds in (6) for some $r \in(0,1)$ and some increasing strictly convex $\Phi$, then

$$
D=e^{i \alpha} D^{*}, \quad D_{0}=D^{*}, \quad f(z)=e^{i \alpha} F\left(e^{i \beta} z\right)
$$

for some real $\alpha$ and $\beta$.

Theorem 2 and its analogue for $M(r,|f|)$ are proved in Section 3.

Theorems 1 and 2 are similar in form to some of the uniqueness results obtained by Jenkins in, e.g., [20]. 
Let $f \in L^{1}(T)$ be real valued, where $T=\{|z|=1\}$, and define

$$
F(z)=\frac{1}{2 \pi} \int_{0}^{2 \pi} f\left(e^{i \varphi}\right) \frac{1+z e^{-i \varphi}}{1-z e^{-i \varphi}} d \varphi .
$$

In [4], Baernstein establishes some sharp bounds on the conjugate function

$$
\tilde{f}\left(e^{i \theta}\right)=\lim _{r \rightarrow 1^{-}} \operatorname{Im} F\left(r e^{i \theta}\right) .
$$

For example, consider the sharp form of Kolmogorov's $L^{p}$-norm inequality

$$
\|\tilde{f}\|_{p} \leqq C_{p}\|f\|_{1} \quad(0<p<1)
$$

with

$$
C_{p}=\|\csc \theta\|_{p}=\left(\frac{1}{2 \pi} \int_{0}^{2 \pi}|\sin \theta|^{-p} d \theta\right)^{1 / p}
$$

due to B. Davis [9]. Baernstein gives an informative proof of (10) in [4], as a limiting, case of a theorem about the analytic functions

$$
F_{\mu}(z)=\int_{T} \frac{1+z e^{-i \varphi}}{1-z e^{-i \varphi}} d \mu\left(e^{i \varphi}\right),
$$

where $\mu \in M(T)$, the class of real valued Borel measures on $T$. We denote by $\|\mu\|$ the total variation of $\mu$, and put

$$
\tilde{\mu}\left(e^{i \theta}\right)=\lim _{r \rightarrow 1^{-}} \operatorname{Im} F_{\mu}\left(r e^{i \theta}\right) .
$$

Then (10) is equivalent to

$$
\|\tilde{\mu}\|_{p} \leqq C_{p}\|\mu\| \quad(0<p<1) .
$$

Equality holds when $\mu$ is the singular measure

$$
v=\frac{1}{2} \delta_{1}-\frac{1}{2} \delta_{-1}
$$

here $\delta_{\zeta} \in M(T)$ is the unit mass concentrated at $\zeta \in T$, so that

$$
F_{v}(z)=\frac{2 z}{1-z^{2}} .
$$

Let us also put, for each $b \in[0,1]$,

$$
v_{b}=\frac{1}{2}(1+b) \delta_{1}-\frac{1}{2}(1-b) \delta_{-1}
$$

so that $\left\|v_{b}\right\|=1, v_{b}(T)=b$. Then Baernstein deduces (13) from his inequality

$$
\int_{0}^{2 \pi}\left|F_{\mu}\left(r e^{i \theta}\right)\right|^{p} d \theta \leqq \int_{0}^{2 \pi}\left|F_{v_{b}}\left(r e^{i \theta}\right)\right|^{p} d \theta \quad(0<r<1 ; 0<p \leqq 2),
$$


valid for all $\mu \in M(T)$ with

$$
\|\mu\| \leqq 1, \quad|\mu(T)|=b .
$$

Concerning (15), we prove

Theorem 3. Assume equality holds in (15) for some $r \in(0,1)$ and some $p \in(0,2]$. Then $\mu$ is a rotation of $v_{b}$, i.e.

$$
\mu(E)=v_{b}\left(e^{i \alpha} E\right) \quad(E \subset T)
$$

for some real $\alpha$. Thus

$$
F_{\mu}(z)=F_{v_{b}}\left(e^{i \alpha} z\right) \quad(|z|<1) .
$$

Suppose now that equality holds in (15) when $r=1$ and $p \in(0,1)$; or, that equality holds in (13) for some $p$. Then we must weaken our conclusions. We give the simpler statement, for (13); recall that one measure giving equality in (13) is $v=v_{0}$.

Theorem 4. Suppose $\mu \in M(T)$ has $\|\mu\|=1$ and satisfies (13) with equality for some $p \in(0,1)$. Then

$$
\mu=\mu_{1}-\mu_{2}
$$

where $\mu_{1}, \mu_{2}$ are positive measures singular with respect to Lebesgue measure such that

and

$$
\mu_{1}(T)=\mu_{2}(T)=\frac{1}{2},
$$

$$
\tilde{\mu}\left(e^{i \theta}\right)=\tilde{v}\left(\omega\left(e^{i \theta}\right)\right)
$$

holds a.e. for some inner function $\omega(z)$ in $U$ with $\omega(0)=0$.

Conversely, given any such $\omega(z)$ there exist positive measures $\mu_{1}$ and $\mu_{2}$, each of mass $1 / 2$, which are singular with respect to Lebesgue measure and mutually singular, such that if $\mu=\mu_{1}-\mu_{2}$ then (16) is valid a.e., and equality holds in (13).

A similar statement (see Theorem 4.2 below) holds for the measures $\mu$ giving equality in (15) when $r=1$ and $p<1$. (This restriction on $p$ is clearly essential, since $F_{v_{b}} \notin H^{1}(U)$.)

Theorems 3 and 4 and some variants, concerning e.g. Davis' sharp form of the weak type $(1,1)$ inequality for conjugate functions $\tilde{f}$, are proved in Sections 4 and 5. Analogues for related problems concerning the $\tilde{f}$ and rearrangements of $f$ are treated in Section 6.

Some results on analogous extremal problems for entire functions have already been given by Baernstein [3], Edrei and Fuchs [11], and the authors [13]. A key step in [13] involves a new property of Baernstein's *-function

$$
u^{*}\left(r e^{i \theta}\right)=\sup _{|E|=2 \theta} \int_{E} u\left(r e^{i \varphi}\right) d \varphi
$$


where $E \subset[-\pi, \pi)$ and $|E|=$ Lebesgue measure of $E$. When $A_{0}$ is an annulus about the origin and $u$ is subharmonic in $A_{0}$, Baernstein [2] has proved that $u^{*}$ is subharmonic in $A_{0} \cap\{0<\arg z<\pi\}$. In [13], we consider the case

$$
u(z)=\log |f(z)|, \quad f(z)=\prod_{n}\left(1-z / z_{n}\right)\left(\sum\left|z_{n}\right|^{-1}<\infty\right),
$$

and prove that harmonicity of $u^{*}$ in the upper half-plane implies $z_{n}=-e^{i \alpha}\left|z_{n}\right|$ for all $n$ and some real $\alpha$, so that $u$ has the key symmetry property

$$
u^{*}\left(r e^{i \theta}\right)=\int_{-\theta}^{\theta} u\left(r e^{i(\alpha+\varphi)}\right) d \varphi \quad(0 \leqq \theta \leqq \pi) .
$$

A basic tool here, Lemma 1.1, yields a similar result for more general subharmonic $u$.

Throughout this paper we systematically refer to the results, methods, terminology and notation of [2] and [4].

\section{Study of subharmonic $u$ having $u^{*}$ harmonic}

Let $u$ be subharmonic on an open set $D$ in the extended plane. The circular symmetrization of $D$ is the open set $D^{*}$ defined as follows: If for $r \in(0, \infty)$ the intersection of $T_{r}=\{|z|=r\}$ with $D$ is $T_{r}$ or the empty set $\emptyset$, then $T_{r} \cap D^{*}$ is to be $T_{r}$ or $\emptyset$, respectively; otherwise $T_{r} \cap D^{*}=\left\{r e^{i \theta}:|\theta|<(2 r)^{-1} \int_{T_{r} \cap D} d \theta\right\}$. Finally, $D^{*}$ contains 0 or $\infty$ if and only if $D$ does. We assume $D^{*}$ is connected.

It is convenient to assume $D \subset\left\{s_{0}<|z|<t_{0}\right\}=A_{0}$ for some $s_{0} \geqq 0, t_{0} \leqq \infty$, and that $u$ is positive in $D$. We put $u \equiv 0$ on $A_{0}-D$ and assume that the extended $u$ is subharmonic on $A_{0}$. Then by [2, p. 141], $u^{*}$ (defined in (17)) is subharmonic on $A_{0} \cap\{\operatorname{Im} z>0\}$.

Under certain conditions [2, p. 153], Baernstein has proved that $u$ harmonic on $D$ implies $u^{*}$ is harmonic on

$$
D_{+}^{*}=D^{*} \cap\{\operatorname{Im} z>0\} .
$$

The following converse statement is a key tool in our proofs.

Lemma 1.1. Let $u$ and $D$ be as described above and suppose that $u^{*}$ is harmonic in $D_{+}^{*}$. Then there exists $\alpha \in[-\pi, \pi)$ such that

$$
\begin{gathered}
D=e^{i \alpha} D^{*} \\
u^{*}\left(r e^{i \theta}\right)=\int_{-\theta}^{\theta} u\left(r e^{i(\alpha+\varphi)}\right) d \varphi=2 \int_{0}^{\theta} u\left(r e^{i(\alpha+\varphi)}\right) d \varphi \quad\left(r e^{i \theta} \in A_{0}, 0 \leqq \theta \leqq \pi\right) .
\end{gathered}
$$

Proof. For each $r \in\left(s_{0}, t_{0}\right)$, we define $v\left(r e^{i \theta}\right)$ for $-\pi \leqq \theta \leqq \pi$ to be the symmetric nonincreasing rearrangement of $u$ restricted to $\{|z|=r\}$ (for an explicit definition of this term, see [2], p. 149). Then

$$
u^{*}\left(r e^{i \theta}\right)=\int_{-\theta}^{\theta} v\left(r e^{i \varphi}\right) d \varphi \quad(0 \leqq \theta \leqq \pi)
$$


so that

$$
\Delta u^{*}\left(r e^{i \theta}\right)=\int_{-\theta}^{\theta} \Delta v\left(r e^{i \varphi}\right) d \varphi \quad\left(r e^{i \theta} \in D_{+}^{*}\right)
$$

(cf. [2, p. 153]). Since $u^{*}$ is harmonic in $D_{+}^{*}, v$ is harmonic in $D^{*}$.

Choose any $r_{1} \in\left(s_{0}, t_{0}\right) \cap D^{*}$ and $\alpha$ real so that

$$
v\left(r_{1}\right)=\max _{|z|=r_{1}} u(z)=u\left(r_{1} e^{i \alpha}\right) .
$$

Then $z_{1}=r_{1} e^{i \alpha} \in D$.

Define

$$
U_{\alpha}\left(r e^{i \theta}\right)=\int_{\alpha-\theta}^{\alpha+\theta} u\left(r e^{i \varphi}\right) d \varphi, \quad V\left(r e^{i \theta}\right)=\int_{-\theta}^{\theta} v\left(r e^{i \varphi}\right) d \varphi,
$$

and choose $\varrho>0$ so that $\left\{\left|z-z_{1}\right| \leqq \varrho\right\} \subset D$. Then

$$
\begin{aligned}
\pi \varrho^{2} u\left(z_{1}\right) & \leqq \iint_{\left|z-z_{1}\right| \leqq \varrho} u=\int_{r_{1}-\varrho}^{r_{1}+\varrho} \int_{\alpha-\theta(r)}^{\alpha+\theta(r)} u\left(r e^{i \varphi}\right) r d \varphi d r \\
& \leqq \int_{r_{1}-\varrho}^{r_{1}+\varrho} u^{*}\left(r e^{i \theta(r)}\right) r d r=\int_{r_{1}-\varrho}^{r_{1}+\varrho} V\left(r e^{i \theta(r)}\right) r d r=\iint_{\left|z-r_{1}\right| \leqq \varrho} v=\pi \varrho^{2} v\left(r_{1}\right) .
\end{aligned}
$$

Since $u\left(z_{1}\right)=v\left(r_{1}\right)$, there is equality throughout. It follows that

$$
U_{\alpha}(z)=u^{*}(z)=V(z)
$$

a.e. on the half-circle $\left\{\left|z-r_{1}\right|=\varrho\right\} \cap D_{+}^{*}$. But $U_{\alpha}-V$ is subharmonic and nonpositive in $D_{+}^{*}$ and vanishes at some interior points of $D_{+}^{*}$. From the maximum principle, we deduce (1.5) throughout $D_{+}^{*}$. Thus (1.1) and the first equality in (1.2) hold.

At this point we may as well suppose $\alpha=0$. It remains to note that (i) for each $r \in\left(s_{0}, t_{0}\right), u\left(r e^{i \theta}\right)$ is nonincreasing on $[0, \pi]$ and nondecreasing on $[-\pi, 0]$, and (ii) $u(\bar{z})=u(z)$ for all $z \in A_{0}$. For, if (i) were false, there would exist an $r$ and $\theta$ such that

$$
u^{*}\left(r e^{i \theta}\right)>\int_{-\theta}^{\theta} u\left(r e^{i \varphi}\right) d \varphi
$$

contradicting (1.5). Thus (i) is true, and then we can use (i) and the same argument to see that (ii) holds.

The same proof yields a useful variant.

Lemma 1.2. Let $u$ and $D$ satisfy the conditions of the first two paragraphs of this section. Let $v$ be harmonic in $D^{*}$ and define $V$ as in (1.4).

If $u^{*} \leqq V$ in $D_{+}^{*}$, and there exists $z_{1}=r_{1} e^{i \alpha} \in D$ such that $u\left(z_{1}\right)=v\left(r_{1}\right)$, then $u^{*}=V$ in $D_{+}^{*}$. In particular, $u^{*}$ is harmonic there.

Lemma 1.2 implies Lemma 1.1 if we choose $v$ to satisfy (1.3). A different choice of $v$ in Lemma 1.2 yields a characterization of the domains for which equality can hold in Baernstein's inequality (5) for Green's functions. For this, see Section 2.

In Section 3 we also need 
Lemma 1.3. Let $u$ and $v$ be Green's functions as described in Theorem 1. Assume there exist $r>0$ and $t \in(-\infty, \infty)$ such that

$$
\min _{\varphi} u\left(r e^{i \varphi}\right)<t<\max _{\varphi} u\left(r e^{i \varphi}\right) .
$$

If for one such pair $(r, t)$,

$$
\int_{-\pi}^{\pi}\left[u\left(r e^{i \varphi}\right)-t\right]^{+} d \varphi=\int_{-\pi}^{\pi}\left[v\left(r e^{i \varphi}\right)-t\right]^{+} d \varphi,
$$

then $u^{*}(z)=v^{*}(z), z \in D_{+}^{*}$.

Proof of Lemma 1.3. Fix $(r, t)$ satisfying the conditions of the lemma, and let $u_{0}=u-t, v_{0}=v-t$. Then

$$
\begin{aligned}
& \int_{-\pi}^{\pi} u_{0}\left(r e^{i \varphi}\right)^{+} d \varphi=\sup _{\varphi} u_{0}^{*}\left(r e^{i \varphi}\right)=u_{0}^{*}\left(r e^{i \theta}\right), \\
& \int_{-\pi}^{\pi} v_{0}\left(r e^{i \varphi}\right)+d \varphi=\sup _{\varphi} v_{0}^{*}\left(r e^{i \psi}\right)=v_{0}^{*}\left(r e^{i \psi}\right)
\end{aligned}
$$

and by assumption $u_{0}^{*}\left(r e^{i \theta}\right)=v_{0}^{*}\left(r e^{i \psi}\right)$. It is a consequence of (1.6) that $0<\theta<\pi$ and $r e^{i \theta} \in D_{+}^{*}$.

We claim that $\theta=\psi$. To see this, we note that $v$ is nonconstant on $\{|z|=r\} \cap D^{*}$; thus $\varphi \rightarrow v\left(r e^{i \varphi}\right)$ cannot be constant on any interval $(\alpha, \beta)$ such that $r e^{i \varphi} \in D^{*}$ when $\alpha<\varphi<\beta$. Thus

$$
v_{0}^{*}\left(r e^{i \varphi}\right)<v_{0}^{*}\left(r e^{i \psi}\right), \quad \varphi \neq \psi
$$

If $\psi \neq \theta$,

$$
v_{0}^{*}\left(r e^{i \psi}\right)=u_{0}^{*}\left(r e^{i \theta}\right) \leqq v_{0}^{*}\left(r e^{i \theta}\right)<v_{0}^{*}\left(r e^{i \psi}\right),
$$

where the first inequality follows from [2, p. 165]. The contradiction shows that $\theta=\psi$ and $u^{*}\left(r e^{i \psi}\right)=v^{*}\left(r e^{i \psi}\right)$. But $u^{*}-v^{*}$ is subharmonic and nonpositive in $D_{+}^{*}$ (cf. [2, Section 7]); since $u^{*}-v^{*}$ vanishes at $r e^{i \psi} \in D_{+}^{*}, u^{*}=v^{*}$ throughout $D_{+}^{*}$, as required.

Our proofs of these lemmas generalize to functions in $n$-space, and lead to uniqueness theorems for certain results of Gariepy-Lewis [15] and BaernsteinTaylor [5]. We intend to describe these extensions in a separate note.

\section{On Green's functions. Proof of Theorem 1}

Let $\Phi$ be as in Theorem 1. Then $\Phi(0+)=\Phi(0)$, and integration by parts yields the representation

$$
\Phi(x)=\Phi(0)+\int_{[0, \infty)}(x-t)^{+} d \mu(t) \quad(0 \leqq x<\infty)
$$


where the measure $\mu$ is defined by $\mu[0, t)=\Phi^{\prime}(t-)(0<t<\infty), \mu\{0\}=\Phi^{\prime}(0+)$; by strict convexity of $\Phi, \mu(J)>0$ for any interval $J \subset[0, \infty)$ of positive length.

Since $u^{*} \leqq v^{*}$ in $\{\operatorname{Im} z \geqq 0\}$ ([2, p. 165]), we have

$$
\int_{-\pi}^{\pi}\left[u\left(r e^{i \varphi}\right)-t\right]^{+} d \varphi \leqq \int_{-\pi}^{\pi}\left[v\left(r e^{i \varphi}\right)-t\right]^{+} d \varphi
$$

for all $r>0$ and real $t$ [2, Proposition 3]. Integrating (2.2) against $d \mu(t)$ over $[0, \infty)$ and using (2.1), we obtain

$$
\int_{-\pi}^{\pi} \Phi\left(u\left(r e^{i \varphi}\right)\right) d \varphi \leqq \int_{-\pi}^{\pi} \Phi\left(v\left(r e^{i \varphi}\right)\right) d \varphi .
$$

Let $r$ be the positive number in $D^{*}$ for which equality in (2.3) has been assumed. Then (2.2) holds with equality a.e. $(d \mu)$ on $0<t<\infty$. Since $\Phi$ is strictly convex on $[0, \infty)$ there is equality in (2.2) for all $t>0$, and thus for all real $t$ since $u$ and $v$ are nonnegative. We conclude, for this $r$,

$$
u^{*}\left(r e^{i \theta}\right)=v^{*}\left(r e^{i \theta}\right) \quad(0 \leqq \theta \leqq \pi) .
$$

(This argument requires that the $\Phi$-means of $u$ and $v$ in (2.3) be finite. When $r=\left|z_{0}\right|$, this is clearly the case if and only if $\int_{0}^{1} \Phi(-\log \varphi) d \varphi=\int_{0}^{\infty} \Phi(x) e^{-x} d x<\infty$.)

Suppose first $z_{0} \neq 0$ or $\infty$. Baernstein has proved [2, p. 166] that

$$
u^{*}(z)=u^{*}(z)+2 \pi \log ^{+}\left|z / z_{0}\right|
$$

is subharmonic in $D_{+}^{*}$ and that $v^{\#}$ is harmonic there. Thus $u^{\#}-v^{\#}$ is subharmonic and nonpositive in $D_{+}^{*}$, and vanishes on $D_{+}^{*} \cap\{|z|=r\}$. It follows that $u^{\#}=v^{\#}$ throughout $D_{+}^{*}$. We conclude from (2.4) that $u^{*}=v^{*}$ in $D_{+}^{*}$, and that $u^{*}$ is harmonic in $D_{1}=D_{+}^{*} \cap\left\{|z|<\left|z_{0}\right|\right\}$ and $D_{2}=D_{+}^{*} \cap\left\{|z|>\left|z_{0}\right|\right\}$.

It follows from Lemma 1.1 that there exists $\alpha_{v} \in \boldsymbol{R}$ such that

$$
\int_{0}^{\theta} u\left(r e^{i\left(\alpha_{v}+\varphi\right)}\right) d \varphi=\int_{0}^{\theta} v\left(r e^{i \varphi}\right) d \varphi, \quad r e^{i \theta} \in D_{v}
$$

for $v=1,2$ and that $u\left(e^{i \alpha_{v}} z\right)=v(z)$ for $z \in D_{v}, v=1,2$. Thus $\alpha_{1}=\alpha_{2}=\alpha$ and $u\left(e^{i \alpha} z\right)=$ $v(z)$ on $D^{*}$.

If $z_{0}=0$ or $\infty$, the argument is simpler since there is no need to introduce $u^{\#}$ or the subdomains $D_{v}$. This concludes the proof of The'orem 1 .

A variant of the above argument gives

Theorem 2.1. Suppose that $u$ and $v$ satisfy the conditions of Theorem 1, except that equality holds in (5) rather than in (3) for some positive $r \in D^{*}, r \neq\left|z_{0}\right|$. Then the conclusion of Theorem 1 still holds. 
Proof. Choose $\alpha$ real so that $u\left(r e^{i \alpha}\right)=M(r, u)$, and let $z_{1}=r e^{i \alpha}$. Then $z_{1} \in D$ and $u\left(z_{1}\right)=v\left(\left|z_{1}\right|\right)$, since $M(r, v)=v(r)$, (cf. [2], pp. 153, 154), and thus the hypotheses of Lemma 1.2 are satisfied with $V=v^{*}$ and $D$ replaced by $D \cap\left\{|z|<\left|z_{0}\right|\right\}$ or $D \cap\left\{|z|>\left|z_{0}\right|\right\}$ according as $r<\left|z_{0}\right|$ or $r>\left|z_{0}\right|$. We conclude that $u^{*}=v^{*}$ in $D_{1}$ or $D_{2}$ respectively, so that $u^{\#}=v^{\#}$ throughout $D_{+}^{*}$. Now the argument used above to complete the proof of Theorem 1 yields the conclusions of that theorem for these $u$ and $v$.

Baernstein has found an inequality like (3) for harmonic measures ([2, Section 8], $[12$, p. 86]) and our methods yield also the corresponding uniqueness result analogous to Theorem 1. Specifically, let $D$ be a domain in $U$, assume $\Gamma=\partial D \cap T$ has positive Lebesgue measure, and let $u$ be the harmonic measure of $\Gamma$ with respect to $D$, i.e., the harmonic function in $D$ whose boundary function is 1 on $\Gamma$ and 0 on $\partial D \cap U$. Denote by $v$ the harmonic measure of $\Gamma^{*}=\partial D^{*} \cap T$ with respect to $D^{*}$, and extend $u$ and $v$ to all of $U$ by setting them equal to zero outside $D$ and $D^{*}$, respectively. Then Baernstein's theorem mentioned above is

$$
\int_{-\pi}^{\pi} \Phi\left(u\left(r e^{i \theta}\right)\right) d \theta \leqq \int_{-\pi}^{\pi} \Phi\left(v\left(r e^{i \theta}\right)\right) d \theta \quad(0<r<1),
$$

for any convex increasing $\Phi$ on $[0,1]$.

The arguments used in this section lead immediately to

Theorem 2.2. If equality holds in (2.5) for some $r \in(0,1) \cap D^{*}$ and a strictly convex $\Phi$, then $D$ is circularly-symmetric with respect to some ray $\arg z=\alpha$.

\section{On convex means of analytic functions. Proof of Theorem 2}

Let $\Phi, f, F, D$ and $D_{0}$ be as in the assumptions of Theorem 2, let $\zeta_{0}=f(0)$ and let $u, v, w$ be Green's functions for $D, D^{*}$ and $D_{0}$ with poles at $\zeta_{0},\left|\zeta_{0}\right|$ and $\left|\zeta_{0}\right|$, respectively. Then Baernstein has shown $[2$, pp. 152, 142, 166] that

$$
\begin{aligned}
& \int_{-\pi}^{\pi} \log \frac{\left|f\left(r e^{i \theta}\right)\right|}{\varrho} d \theta-2 \pi \log \frac{|f(0)|}{\varrho} \\
\leqq & \int_{-\pi}^{\pi}\left[u\left(\varrho e^{i \varphi}\right)+\log r\right]^{+} d \varphi \leqq \int_{-\pi}^{\pi}\left[v\left(\varrho e^{i \varphi}\right)+\log r\right]^{+} d \varphi \\
\leqq & \int_{-\pi}^{\pi}\left[w\left(\varrho e^{i \varphi}\right)+\log r\right]^{+} d \varphi \\
= & \int_{-\pi}^{\pi} \log ^{+} \frac{\left|F\left(r e^{i \theta}\right)\right|}{\varrho} d \theta-2 \pi \log ^{+} \frac{|f(0)|}{\varrho}
\end{aligned}
$$


holds for all $\varrho>0$ and $0<r<1$; as usual, we set $u, v$ and $w$ equal to zero outside of $D, D^{*}$ and $D_{0}$, respectively.

The same argument used for (2.1) yields also

$$
\Phi(x)=\Phi(\tau)+(x-\tau) \Phi^{\prime}(\tau-)+\int_{[\tau, \infty)}(x-t)^{+} d \mu(t) \quad(-\infty<\tau<x)
$$

with $\mu(-\infty, t)=\Phi^{\prime}(t-)$. Thus if $\tau<\inf (x, y)$ and we put $t=\log \varrho$,

$$
\Phi(x)-\Phi(y)=(x-y) \Phi^{\prime}(\tau-)+\int_{\eta}^{\infty}[x-\log \varrho]^{+} d v(\varrho)-\int_{\eta}^{\infty}[y-\log \varrho]^{+} d v(\varrho)
$$

where $\eta=\exp \tau, v(0, \varrho)=\mu(-\infty, \log \varrho)$ and we interpret $\int_{\eta}^{\infty}=\int_{[\eta, \infty)}$. Let $\tau \rightarrow-\infty$ in (3.2), choose $x=\log \left|F\left(r e^{i \theta}\right)\right|$ and $y=\log \left|f\left(r e^{i \theta}\right)\right|$ there, and integrate the result over $[-\pi, \pi]$ to obtain

where

$$
\begin{gathered}
\int_{-\pi}^{\pi} \Phi\left(\log \left|F\left(r e^{i \theta}\right)\right|\right) d \theta-\int_{-\pi}^{\pi} \Phi\left(\log \left|f\left(r e^{i \theta}\right)\right|\right) d \theta \\
=I_{0} \Phi^{\prime}(-\infty)+\lim _{\eta \rightarrow 0+} \int_{\eta}^{\infty} I(\varrho) d v(\varrho)
\end{gathered}
$$

$$
\begin{gathered}
I_{0}=\int_{-\pi}^{\pi} \log \left|F\left(r e^{i \theta}\right)\right| d \theta-\int_{-\pi}^{\pi} \log \left|f\left(r e^{i \theta}\right)\right| d \theta, \\
I(\varrho)=\int_{-\pi}^{\pi} \log \frac{\left|F\left(r e^{i \theta}\right)\right|}{\varrho} d \theta-\int_{-\pi}^{\pi} \log \frac{\left|f\left(r e^{i \theta}\right)\right|}{\varrho} d \theta .
\end{gathered}
$$

By Baernstein's inequality (6), $I_{0} \geqq 0$ and $I(\varrho) \geqq 0$.

Now assume $r \in(0,1)$ is such that equality holds in (6). Then from (3.3), $d v \geqq 0$, and the fact that $\Phi^{\prime}(-\infty) \geqq 0$,

$$
I_{0} \Phi^{\prime}(-\infty)=0, \quad \int_{0}^{\infty} I(\varrho) d v(\varrho)=0 .
$$

Since $\Phi$ is strictly convex, $v(J)>0$ for all intervals $J \subset(0, \infty)$ of positive length; since $I(\varrho)$ is continuous, (3.4) implies

$$
I(\varrho)=0 \quad(0<\varrho<\infty) .
$$

We conclude from (3.1) that

$$
\int_{-\pi}^{\pi}\left[u\left(\varrho e^{i \varphi}\right)+\log r\right]^{+} d \varphi=\int_{-\pi}^{\pi}\left[v\left(\varrho e^{i \varphi}\right)+\log r\right]^{+} d \varphi=\int_{-\pi}^{\pi}\left[w\left(\varrho e^{i \varphi}\right)+\log r\right]^{+} d \varphi
$$

for all $\varrho>0$.

It is clear from (3.6) that $D_{0}=D^{*}$, since otherwise $v(\zeta)<w(\zeta)$ for all $\zeta \in D^{*}$, $\zeta \neq\left|\zeta_{0}\right|$ 
We claim that $u^{*}=v^{*}$. This is a consequence of Lemma 1.3 and (3.6), provided there exists a $\varrho>0$ such that

$$
\inf _{\varphi} u\left(\varrho e^{i \varphi}\right)<-\log r<\sup _{\varphi} u\left(\varrho e^{i \varphi}\right) .
$$

Let

$$
D_{r}=\{u(\zeta)>-\log r\}, \quad E_{r}=\{v(\zeta)>-\log r\},
$$

and suppose there exists some $\varrho_{0}>0$ such that $|\zeta|=\varrho_{0}$ intersects both $D_{r}$ and $C-D_{r}$. Then certainly

$$
\mathfrak{M}\left(\varrho_{0}, u\right) \equiv \inf _{\varphi} u\left(\varrho_{0} e^{i \varphi}\right) \leqq-\log r<\sup _{\varphi} u\left(\varrho_{0} e^{i \varphi}\right),
$$

and if $\mathfrak{M}\left(\varrho_{0}, u\right)=0$ then (3.7) holds with $\varrho=\varrho_{0}$. Otherwise the circle $|\zeta|=\varrho_{0}$ is contained in $D$, so that $\mathfrak{M}(\varrho, u)$ is a nonconstant continuous function near $\varrho_{0}$ and thus (3.7) must hold for some $\varrho$ near $\varrho_{0}$. We conclude that $u^{\#}$ (cf. (2.4)) is harmonic on $D_{+}^{*}$ and, by Lemma 1.1 ,

$$
u(\zeta)=v\left(e^{-i \alpha} \zeta\right) \quad \text { and } \quad D=e^{i \alpha} D^{*}
$$

where $\alpha$ is a real constant $\left(=\arg \zeta_{0}\right.$ if $\left.\zeta_{0} \neq 0\right)$.

It remains to consider the case when every circle $|\zeta|=\varrho$ is either contained in $D_{r}$ or disjoint from it. Since $D_{r}$ is connected, $D_{r}=\left\{|\zeta|<\varrho_{1}\right\}$ or $D_{r}=\left\{\varrho_{1}<|\zeta|<\varrho_{2}\right\}$. In the latter case, we must have $\left\{|\zeta| \leqq \varrho_{1}\right\} \subset D$ since $D^{*}=D_{0}$ is known to be simply connected; then $\mathfrak{M}(\varrho, u)$ is strictly decreasing on $\left(0, \varrho_{2}\right)$ and thus

$$
-\log r \geqq u(0)>\mathfrak{M}(\varrho, u) \quad\left(\varrho_{1}<\varrho<\varrho_{2}\right),
$$

a contradiction. We deduce that $D_{r}=\left\{|\zeta|<\varrho_{1}\right\}$, and claim that (3.6) implies $E_{r}=D_{r}$. In fact, by (3.6)

$$
\int_{-\pi}^{\pi}\left[v\left(\varrho e^{i \varphi}\right)+\log r\right]^{+} d \varphi=\int_{-\pi}^{\pi}\left[u\left(\varrho e^{i \varphi}\right)+\log r\right]^{+} d \varphi>0
$$

if and only if $0 \leqq \varrho<\varrho_{1}$, so that $E_{r} \subset D_{r}$. Further, if $\zeta \in D_{r}-E_{r}$ then (3.6) implies

$$
\int_{-\pi}^{\pi}\left[u\left(\varrho e^{i \varphi}\right)+\log r\right] d \varphi>\int_{-\pi}^{\pi}\left[v\left(\varrho e^{i \varphi}\right)+\log r\right] d \varphi
$$

for $|\zeta|<\varrho<\varrho_{1}$, and this contradicts Baernstein's inequality (3). Thus in this case $u_{0}=u+\log r$ and $v_{0}=v+\log r$ are Green's functions for $\left\{|\zeta|<\varrho_{1}\right\}$ with poles at $\zeta_{0}$ and $\left|\zeta_{0}\right|$ respectively, so that again (3.8) holds.

Finally we claim that $f(z)=e^{i \alpha} F\left(e^{i \beta} z\right)$ for some real $\beta$. We know that $f(U)=$ $e^{i \alpha} D_{0}$ and $f(0)=e^{i \alpha} F(0)$. Define

$$
\omega(z)=F^{-1}\left(e^{-i \alpha} f(z)\right) \quad(z \in U) ;
$$

by Schwarz' lemma, we have $\omega(z)=e^{i \beta} z$ or $|\omega(z)|<|z|$ in $\{0<|z|<1\}$. To show that the latter possibility cannot occur, suppose it does and let $h$ be the harmonic 
function in $\{|z|<r\}$ whose boundary values are $\Phi\left(\log \left|F\left(r e^{i \theta}\right)\right|\right)$. Then we have

$$
\begin{aligned}
& \int_{-\pi}^{\pi} \Phi\left(\log \left|f\left(r e^{i \theta}\right)\right|\right) d \theta=\int_{-\pi}^{\pi} \Phi\left(\log \left|F\left(\omega\left(r e^{i \theta}\right)\right)\right|\right) d \theta \\
& <\int_{-\pi}^{\pi} h\left(\omega\left(r e^{i \theta}\right)\right) d \theta=2 \pi h(\omega(0))=\int_{-\pi}^{\pi} h\left(r e^{i \theta}\right) d \theta \\
& =\int_{-\pi}^{\pi} \Phi\left(\log \left|F\left(r e^{i \theta}\right)\right|\right) d \theta,
\end{aligned}
$$

the strict inequality holding because $\Phi(\log |F(z)|)$ is strictly subharmonic (i.e. not harmonic) throughout $\{|z|<r\}$, due to the strict convexity of $\Phi$. But (3.10) is inconsistent with our hypothesis of equality in (6), and the proof of Theorem 2 is complete.

Now choose $\Phi(x)=\exp (p x)$ in (6), take $p$ th roots and let $p \rightarrow \infty$ to obtain

$$
M(r,|f|) \leqq M(r,|F|) \quad(0<r<1) .
$$

Theorem 3.1. Let $f$ and $F$ be as in Theorem 2. If equality holds in (3.11) for some $r \in(0,1)$, then (7) is true for some real $\alpha$ and $\beta$.

Proof. Using (3.2) with $\Phi(x)=\exp (p x), \tau=-\infty$ and $x=\log \left|f\left(r e^{i \theta}\right)\right|, y=-\infty$ we see that

Thus (3.1) implies

$$
\left|f\left(r e^{i \theta}\right)\right|^{p}=\int_{0}^{\infty} \log ^{+} \frac{\left|f\left(r e^{i \theta}\right)\right|}{\varrho} p^{2} \varrho^{p-1} d \varrho .
$$

$$
\begin{aligned}
& \int_{-\pi}^{\pi}\left|f\left(r e^{i \theta}\right)\right|^{p} d \theta-2 \pi|f(0)|^{p} \leqq p^{2} \int_{0}^{\infty} \varrho^{p-1} d \varrho \int_{-\pi}^{\pi}\left[u\left(\varrho e^{i \varphi}\right)+\log r\right]^{+} d \varphi \\
\leqq & p^{2} \int_{0}^{\infty} \varrho^{p-1} d \varrho \int_{-\pi}^{\pi}\left[v\left(\varrho e^{i \varphi}\right)+\log r\right]^{+} d \varphi \\
\leqq & p^{2} \int_{0}^{\infty} \varrho^{p-1} d \varrho \int_{-\pi}^{\pi}\left[w\left(p e^{i \varphi}\right)+\log r\right]^{+} d \varphi=\int_{-\pi}^{\pi}\left|F\left(r e^{i \theta}\right)\right|^{p} d \theta-2 \pi|f(0)|^{p}
\end{aligned}
$$

where $u, v$ and $w$ are the Green's functions introduced at the start of this section.

Let $r$ be the fixed value for which equality holds in (3.11). Taking $p$ th roots and letting $p \rightarrow \infty$ in (3.12), we obtain a chain of equalities. To describe the limiting relations, we note that if $\psi \in L^{1}(0, \infty)$ is nonnegative with $\int_{0}^{\infty} \psi>0$ and, to fix the ideas, compact support in $[0, \infty)$, then

$$
\lim _{p \rightarrow \infty}\left\{\int_{0}^{\infty} \varrho^{p-1} \psi(\varrho) d \varrho\right\}^{1 / p}=\sup \left\{\varrho: \int_{\varrho}^{\infty} \psi>0\right\} .
$$


Thus we deduce from (3.12) that

$$
\begin{aligned}
\varrho_{0}=\sup \left\{\varrho: \int_{-\pi}^{\pi}\left[u\left(\varrho e^{i \varphi}\right)+\log r\right]^{+} d \varphi>0\right\} & =\sup \left\{\varrho: \int_{-\pi}^{\pi}\left[v\left(\varrho e^{i \varphi}\right)+\log r\right]^{+} d \varphi>0\right\} \\
& =\sup \left\{\varrho: \int_{-\pi}^{\pi}\left[w\left(\varrho e^{i \varphi}\right)+\log r\right]^{+} d \varphi>0\right\} .
\end{aligned}
$$

We conclude that $M(\varrho, u)=\max _{\varphi} u\left(\varrho e^{i \varphi}\right)$ satisfies

$$
M\left(\varrho_{0}, u\right)=M\left(\varrho_{0}, v\right)=M\left(\varrho_{0}, w\right)=-\log r .
$$

It follows that $D^{*}=D_{0}$, for otherwise $v(\zeta)<w(\zeta)$ throughout $D_{0}-\left\{\left|\zeta_{0}\right|\right\}$ and (3.13) would be violated. Now we apply Theorem 2.1, and deduce $D=e^{i \alpha} D^{*}$ and $u(z)=v\left(e^{-i \alpha} z\right)$

To complete the proof of (7), we define $\omega(z)$ as in (3.9) and observe that our hypothesis

$$
M(r,|f|)=M(r,|F \circ \omega|)=M(r,|F|)
$$

implies $\left|\omega\left(r e^{i \theta}\right)\right|=r$ for some $\theta$. Thus, $\omega(z)=e^{i \beta} z$ for some real $\beta$, and we are done.

\section{Some extensions of Theorems 3 and 4}

Before proceeding to the proofs of Theorems 3 and 4, we state some related results from [4] and describe our corresponding uniqueness theorems.

Let $\mu \in M(T)$ and $F_{\mu}$ be as defined in (11), let $v_{b}$ be the extremal measure given in (14), and put

$$
G_{b}(z)=F_{v_{b}}(z) \quad(z \in U, 0 \leqq b \leqq 1) .
$$

We suppose throughout that

Then if

$$
\mu(T) \geqq 0 .
$$

$$
\|\mu\| \leqq 1, \quad b=\mu(T)
$$

and $0<r<1$, Baernstein has proved [4]

$$
\begin{gathered}
\int_{0}^{2 \pi}\left|F_{\mu}\left(r e^{i \theta}\right)\right|^{p} d \theta \leqq \int_{0}^{2 \pi}\left|G_{b}\left(r e^{i \theta}\right)\right|^{p} d \theta \quad(0<p \leqq 2), \\
\int_{0}^{2 \pi}\left|\operatorname{Im} F_{\mu}\left(r e^{i \theta}\right)\right|^{p} d \theta \leqq \int_{0}^{2 \pi}\left|\operatorname{Im} G_{b}\left(r e^{i \theta}\right)\right|^{p} d \theta \quad(1 \leqq p \leqq 2), \\
\int_{0}^{2 \pi}\left|\operatorname{Re} F_{\mu}\left(r e^{i \theta}\right)\right|^{p} d \theta \leqq \int_{0}^{2 \pi}\left|\operatorname{Re} G_{b}\left(r e^{i \theta}\right)\right|^{p} d \theta \quad(1<p<\infty) .
\end{gathered}
$$

Our first extension of Theorem 3 is 
Theorem 4.1. Let $\mu$ satisfy (4.1) and assume equality holds in one of (4.2a)(4.2c) for some $r \in(0,1)$ and one value of $p$ in the associated range. Then

$$
\begin{array}{cc}
F_{\mu}(z)=G_{b}\left(e^{i \alpha} z\right) & (|z|<1), \\
\mu(E)=v_{b}\left(e^{i \alpha} E\right) & (E \subset T)
\end{array}
$$

for some real $\alpha$.

Inequality (4.2c) obviously holds for $p=1$ as well, by continuity; but the corresponding uniqueness statement requires some care. In case

$$
b=\mu(T)=F(0)>0
$$

and $r \in(0,1)$ is such that $\operatorname{Re} F_{\mu}\left(r e^{i \theta}\right) \geqq 0$ for all $\theta$, then equality in $(4.2 \mathrm{c})$ with $p=1$ implies $\operatorname{Re} G_{b}\left(r e^{i \theta}\right) \geqq 0$ [i.e. $\left.r \leqq r_{b} \equiv b /\left(1+\left(1-b^{2}\right)^{1 / 2}\right)\right]$, and obviously nothing more can be said. Our proof does give, however:

Let $\mu$ satisfy (4.1) and suppose

$$
\int_{0}^{2 \pi}\left|\operatorname{Re} F_{\mu}\left(r e^{i \theta}\right)\right| d \theta=\int_{0}^{2 \pi}\left|\operatorname{Re} G_{b}\left(r e^{i \theta}\right)\right| d \theta
$$

for some $r \in\left(r_{b}, 1\right)$. Then (4.3), (4.4) still hold.

Since the means in (4.5) fail to be strictly convex, our treatment of this problem will indicate how our earlier theorems involving convex integral means can be slightly generalized. In this case we are concerned with means $\int \Phi\left(\operatorname{Re} F_{\mu}\right)$ where $\Phi(x)=|x|$ is linear except near 0 , and we have to assume

$$
\min _{\theta} \operatorname{Re} F_{\mu}\left(r e^{i \theta}\right)<0<\max _{\theta} \operatorname{Re} F_{\mu}\left(r e^{i \theta}\right)
$$

compare hypothesis (1.6) in Lemma 1.3.

Since $F_{\mu} \in H^{p}(U)$ for all $p<1 \quad([10]$, pp. 2,35$)$, the boundary values

$$
F_{\mu}\left(e^{i \theta}\right)=\lim _{r \rightarrow 1} F_{\mu}\left(r e^{i \theta}\right)
$$

exist a.e. and (4.2a) still holds with $r=1$ and $0<p<1$. We shall prove

Theorem 4.2. Suppose $\mu \in M(T)$ satisfies $\|\mu\| \leqq 1$ and

$$
\int_{0}^{2 \pi}\left|F_{\mu}\left(e^{i \theta}\right)\right|^{p} d \theta=\int_{0}^{2 \pi}\left|G_{b}\left(e^{i \theta}\right)\right|^{p} d \theta
$$

for some $p \in(0,1)$ and some nonnegative $b \leqq \mu(T)$. Then $\mu(T)=b,\|\mu\|=1$ and

$$
\mu=\mu_{+}-\mu_{-}
$$

where $\mu_{+}, \mu_{-}$are positive measures singular with respect to Lebesgue measure and having

$$
\mu_{+}(T)=\frac{1}{2}(1+b), \quad \mu_{-}(T)=\frac{1}{2}(1-b) .
$$


Further,

$$
F_{\mu}(z)=G_{b}(\omega(z)) \quad(|z|<1)
$$

for some inner function $\omega(z)$ in $U$ with $\omega(0)=0$.

Conversely, given any such $\omega(z)$ and any $b \in[0,1]$, then there exist positive measures $\mu_{+}$and $\mu_{-}$which are singular with respect to Lebesgue measure and mutually singular, such that if $\mu=\mu_{+}-\mu_{-}$then (4.6)-(4.8) all hold.

It is easy to see that the case $b=0$ of Theorem 4.2 contains Theorem 4.

Baernstein deduces $(4.2 \mathrm{a})-(4.2 \mathrm{c})$ from an interesting result for analytic functions not necessarily of the form $F_{\mu}$. Following Section 4 in [4], assume $Q$ is a bounded domain containing 0 and Steiner-symmetric with respect to $R$ ([16], p. 68). Determine $B>0$ by $Q \cap\{\operatorname{Re} z=0\}=(-i B, i B)$, and define the subdomains

$$
Q_{\beta}=Q-\{i y: \beta \leqq|y|<B\} \quad(0<\beta \leqq B) .
$$

For each real $b \in Q$ and $\beta \in(0, B]$, let $H_{\beta, b}$ be the univalent map of $U$ onto $Q_{\beta}$ with $H_{\beta, b}(0)=b$ and $H_{\beta, b}^{\prime}(0)>0$. Actually, there is no loss of generality in assuming throughout, as we shall, that

$$
b \geqq 0 \text {. }
$$

To cover the limiting case $\beta=0$, define $H_{0, b}$ for positive $b \in Q$ to be the conformal map from $U$ onto $Q \cap\{\operatorname{Re} w>0\}$ having $H_{0, b}(0)=b$ and $H_{0, b}^{\prime}(0)>0$; and put $H_{0,0}(z) \equiv 0$.

For all nonnegative $b \in Q$ and $\beta \in[0, B]$, define $\mathfrak{F}(\beta, b)$ to be the class of all analytic functions $F$ with

$$
F: U \rightarrow Q, \quad F(0)=b
$$

$$
\int_{0}^{2 \pi}\left|\operatorname{Re} F\left(e^{i \theta}\right)\right| d \theta \leqq \int_{0}^{2 \pi}\left|\operatorname{Re} H_{\beta, b}\left(e^{i \theta}\right)\right| d \theta .
$$

With these assumptions, Baernstein proves:

For all $F \in \mathfrak{F}(\beta, b)$ and $0<r<1$,

$$
\begin{gathered}
\int_{0}^{2 \pi}\left|F\left(r e^{i \theta}\right)\right|^{p} d \theta \leqq \int_{0}^{2 \pi}\left|H_{\beta, b}\left(r e^{i \theta}\right)\right|^{p} d \theta \quad(0<p \leqq 2), \\
\int_{0}^{2 \pi}\left|\operatorname{Im} F\left(r e^{i \theta}\right)\right|^{p} d \theta \leqq \int_{0}^{2 \pi}\left|\operatorname{Im} H_{\beta, b}\left(r e^{i \theta}\right)\right|^{p} d \theta \quad(1 \leqq p \leqq 2), \\
\int_{0}^{2 \pi}\left|\operatorname{Re} F\left(r e^{i \theta}\right)\right|^{p} d \theta \leqq \int_{0}^{2 \pi}\left|\operatorname{Re} H_{\beta, b}\left(r e^{i \theta}\right)\right|^{p} d \theta \quad(1 \leqq p \leqq \infty) .
\end{gathered}
$$

In order to formulate this result as the solution of an extremal problem, Baernstein observes that

$$
\varphi(\beta)=\frac{1}{2 \pi} \int_{0}^{2 \pi}\left|\operatorname{Re} H_{\beta, b}\left(e^{i \theta}\right)\right| d \theta \quad(0 \leqq \beta \leqq B)
$$


is, for fixed nonnegative $b \in Q$, strictly increasing on $[0, B]$; further, $\varphi(0)=b$ and, for each $F$ satisfying (4.9), there exists a unique $\beta \in[0, B]$ such that

$$
\varphi(\beta)=\frac{1}{2 \pi} \int_{0}^{2 \pi}\left|\operatorname{Re} F\left(e^{i \theta}\right)\right| d \theta .
$$

Thus, for the problem of maximizing the integral means in (4.11) among all $F$ in the subclass

$$
\tilde{\widetilde{F}}(\beta, b)=\{F \in \mathfrak{F}(\beta, b): \text { equality holds in }(4.10)\},
$$

$H_{\beta, b}$ is the unique extremal in

$$
\left\{H_{t, b}: 0 \leqq t \leqq B\right\} \cap \tilde{\widetilde{F}}(\beta, b) .
$$

We now consider to what extent this extremal is unique in the full class $\mathfrak{F}(\beta, b)$.

Theorem 4.3. Given any $F \in \mathfrak{F}(\beta, b)$, the inequalities (4.11) are all strict, for each $r \in(0,1)$ and admissible $p$, unless

$$
F(z)=H_{\beta, b}\left(e^{i \alpha} z\right) \quad(|z|<1)
$$

for some real $\alpha$.

There is one gloss here: we do not regard as admissible the choice $p=1$ in (4.11c) for any $r \in(0,1)$ such that

$$
\operatorname{Re} F\left(r e^{i \theta}\right) \geqq 0 \quad \text { and } \quad \operatorname{Re} H_{\beta, b}\left(r e^{i \theta}\right) \geqq 0 \quad(0 \leqq \theta \leqq 2 \pi) .
$$

There is a variant of Theorem 4.3 concerning the $F \in \mathfrak{F}(\beta, b)$ for which equality holds in (4.11a) or (4.11b) for $r=1$ and some appropriate $p$. It will be clear from the proofs of Theorems 4.2 and 6.2 how to state and prove this variant.

In [4, Section 2], Baernstein also gives a function theoretic proof of Davis' sharp form of Kolmogorov's weak type $(1,1)$ inequality for conjugate functions. To state this, let $f \in L^{1}(T)$ be real valued and define $\tilde{f}$ by (9). Put

$$
E_{t}(\tilde{f})=\left\{e^{i \theta}:\left|\tilde{f}\left(e^{i \theta}\right)\right| \geqq t\right\},
$$

and for measurable $E \subset T$ let us also put $|E|=$ Lebesgue measure of $E$. Then Davis inequality is

$$
\left|E_{:}(\tilde{f})\right| \leqq 2 \pi \Theta t^{-1}\|f\|_{1} \quad(0<t<\infty)
$$

where

$$
\Theta^{-1}=\left\|\operatorname{Re} G\left(e^{i \theta}\right)\right\|_{1}, \quad G(z)=\frac{2}{\pi} \log \left(\frac{1+z}{1-z}\right) .
$$

Theorem 4.4. Suppose $f$ satisfies (4.13) with equality for some $t>0$ and $f \not \equiv 0$. Then

$$
f\left(e^{i \theta}\right)=t \operatorname{Re} G\left(\omega\left(e^{i \theta}\right)\right)
$$

for some inner function $\omega$ on $U$ having $\omega(0)=0$. 
Conversely, every such $f$ satisfies

for this $t$.

$$
\left|E_{t}(\tilde{f})\right|=2 \pi, \quad\|f\|_{1}=t \Theta^{-1}
$$

In particular, there never is equality in (4.13) if $\int_{T} f \neq 0$.

We proceed to prove Theorems 4.2 and 4.4; the proofs of Theorems 4.1 and 4.3 will be given in Section 5 .

Proof of Theorem 4.2. We start from the elegant formula

$$
\int_{0}^{2 \pi}\left|F\left(r e^{i \theta}\right)\right|^{p} d \theta=p^{2} \int_{-\infty}^{\infty} \int_{-\infty}^{\infty} N(r, w, F)|w|^{p-2} d u d v+2 \pi|F(0)|^{p}
$$

used in [4] (cf. [16], p. 42), and valid for all $F$ analytic in $U$, say. Here $0<r<1$, $0<p<\infty, w=u+i v$ and $N(r, w, F)$ is the usual Nevanlinna counting function for the value $w$ of $F[17$, p. 6].

We shall need (4.14) also for $r=1$. Let us write

$$
F=F_{\mu}, \quad G=G_{b}=F_{v_{b}}
$$

and recall that $F \in H^{p}(U)$ for $p<1$ since $\mu \in M(T)$ ([10], pp. 2, 35). Thus $F$ is of bounded characteristic and so by Jensen's theorem

$$
N(1, w, F)=\lim _{r \rightarrow 1} N(r, w, F) \leqq B(w)
$$

where

$$
B(w)=\frac{1}{2 \pi} \int_{0}^{2 \pi} \log \left|\frac{w-F\left(e^{i \theta}\right)}{w-F(0)}\right| d \theta
$$

is uniformly bounded except near $F(0)$, and $B(w) \rightarrow 0$ uniformly when $|w| \rightarrow \infty$.

On $0<r<1,\{N(r, w, F)+\log |w-F(0)|\}$ is an increasing family of functions subharmonic (by Jensen's theorem) and continuous in $\boldsymbol{C}$. Thus, $N(1, w, F)$ inherits lower semicontinuity and the subharmonic mean value property from $\{N(r, w, F)\}$, and subharmonicity of $N(1, w, F)$ is a question of continuity. In any case, the upper regularization

$$
q(w)=\limsup _{\zeta \rightarrow w} N(1, \zeta, F)
$$

of the limit (4.15) is subharmonic throughout $C-\{F(0)\}$, with

$$
q(w)=N(1, w, F) \quad(w \in C-\mathscr{E})
$$

where $\mathscr{E}$ has capacity zero,

$$
N(1, w, F) \leqq q(w)
$$


in $C$ ([19], Theorems 7.39 and 7.33); and $q(w)+\log |w-F(0)|$ is subharmonic throughout $C$. Thus, by (4.17),

is continuous in $\{v \geqq 0\}$.

$$
\int_{-v}^{v} q(u+i s) d s
$$

We shall need some basic results on ${ }^{*}$-functions from Section 5 of [4]. If

$$
N^{*}(r, w, F)=\sup _{|E|=2 v} \int_{E} N(r, u+i s, F) d s \quad(v \geqq 0)
$$

then for $r<1$

$$
N^{\#}(r, w, F)=N^{*}(r, w, F)+2 \pi[u-\operatorname{Re} F(0)]^{+}
$$

is subharmonic in $\{v>0\}$ and continuous in $\{v \geqq 0\}$. By (4.15), the definition (4.19) remains valid when $r=1$; because of (4.17) we can define $q^{*}(w)$ in the same way. Further, it is easy to see that

$$
q^{*}(w)=N^{*}(1, w, F)=\lim _{r \rightarrow 1} N^{*}(r, w, F) \quad(v \geqq 0) .
$$

We observe also that

$$
q^{\#}(w)=q^{*}(w)+2 \pi[u-\operatorname{Re} F(0)]^{+}
$$

is subharmonic in $\{v>0\}$ and continuous in $\{v \geqq 0\}$. To see this, notice that for each $\varepsilon>0$ the function $q_{\varepsilon}(w)=[q(w)-\varepsilon]^{+}$has compact support, so that

$$
q_{\varepsilon}^{\#}(w)=q_{\varepsilon}^{*}(w)+2 \pi[u-\operatorname{Re} F(0)]^{+}
$$

has the subharmonicity and continuity properties claimed for $q^{\#}$ (cf. [4], Section 5). Now our assertion for $q^{\#}$ follows from the fact that the majorants

decrease to $q^{\#}(w)$ when $\varepsilon \downarrow 0$.

$$
Q_{\varepsilon}(w)=q_{\varepsilon}^{\#}(w)+2 v \varepsilon
$$

These considerations lead to two lemmas, analogues of Lemmas 1.1 and 1.2, which will be needed in Sections 5 and 6. Motivated by the assumptions of Section 1 , let $p(w)$ be positive on an open set $D \subset B_{0}=\left\{w=u+i v: \xi_{0}<u<\tau_{0}\right\}$, put $p=0$ on $B_{0}-D$, and suppose the extended $p$ is subharmonic on $B_{0}$. Assume further that

$$
\lim _{v \rightarrow \pm \infty} p(u+i v)=0 \quad\left(\xi_{0}<u<\tau_{0}\right)
$$

uniformly on compact subintervals of $\left(\xi_{0}, \tau_{0}\right)$, and define $p^{*}(w)$ as in (4.19). Let $D^{*}$ be the Steiner symmetrization (with respect to $R$ ) of $D$, and let $D_{+}^{*}=D^{*} \cap\{v>0\}$. Then by the remarks in the previous paragraph, $p^{*}$ is subharmonic on $B_{0} \cap\{v>0\}$ and continuous on $B_{0} \cap\{v \geqq 0\}$.

In this setting, Lemmas 1.1 and 1.2 have exact analogues which we shall refer to as Lemmas 4.1 and 4.2 respectively. The proof of Lemma 4.1 (and its slight variant for Lemma 4.2) is the same as that for Lemma 1.1 (except that rectangular rather than polar coordinates are used). Let us explicitly state only the first of the two lemmas. 
Lemma 4.1. Let $p(w)$ and $D$ be as described above and suppose that $p^{*}(w)$ is harmonic in $D_{+}^{*}$. Then there exists $\alpha \in \boldsymbol{R}$ such that

$$
\begin{gathered}
D=D^{*}+i \alpha, \\
p^{*}(w)=\int_{\alpha-v}^{\alpha+v} p(u+i s) d s=2 \int_{\alpha}^{\alpha+v} p(u+i s) d s \quad\left(w=u+i v \in B_{0}, v \geqq 0\right) .
\end{gathered}
$$

Consider now the function $G=G_{b}$ of Theorern 4.2. From the discussion in [4], Sections 3-5, $G$ is univalent and $N(1, w, G)$ is Green's function of the Steiner symmetric domain $G(U)$, with pole at $G(0)=b$. In particular, $N(1, w, G)+\log |w-b|$ is harmonic in $G(U)$ and, for all real $u, v \rightarrow N(1, u+i v, G)$ is nonincreasing on $0<v<\infty$ and even; it follows that

$$
N^{\#}(1, w, G)=\int_{-v}^{v} N(1, u+i s, G) d s+2 \pi[u-b]^{+}
$$

is harmonic in $G(U)^{+}=G(U) \cap\{v>0\}$. We need Baernstein's key inequality

$$
N^{*}(r, w, F) \leqq N^{*}(r, w, G) \quad(0<r<1 ; v \geqq 0) .
$$

Recall that we can let $r \rightarrow 1$ here and obtain

$$
N^{*}(1, w, F) \leqq N^{*}(1, w, G) \quad(v \geqq 0) .
$$

After these preliminaries, we seek consequences of our assumption (4.6). In the first place, we must have $b=\mu(T)$ and $\|\mu\|=1$. For, if we put $\mu_{1}=\mu_{i}^{\prime}\|\mu\|$, then $\left\|\mu_{1}\right\|=1$ and $c=\mu_{1}(T)$ satisfies, by assumption, $c \geqq \mu(T) \geqq b$. If $c>b$,

$$
\|\mu\|^{-p} \int_{0}^{2 \pi}\left|F_{\mu}\left(e^{i \theta}\right)\right|^{p} d \theta=\int_{0}^{2 \pi}\left|F_{\mu_{1}}\left(e^{i \theta}\right)\right|^{p} d \theta \leqq \int_{0}^{2 \pi}\left|G_{c}\left(e^{i \theta}\right)\right|^{p} d \theta<\int_{0}^{2 \pi}\left|G_{b}\left(e^{i \theta}\right)\right|^{p} d \theta
$$

by Baernstein's inequality (4.2a) and the fact that

$$
\psi(b)=\int_{0}^{2 \pi}\left|G_{b}\left(e^{i \theta}\right)\right|^{p} d \theta=2 \int_{0}^{\pi}\left(\frac{1+b \cos \theta}{\sin \theta}\right)^{p} d \theta
$$

is strictly decreasing on $0 \leqq b \leqq 1$ when $p<1$. Since (4.23) contradicts (4.6), we have shown $b=\mu(T)$; another look at (4.23) yields $\|\mu\|=1$.

Recalling (4.14), the fact that $F(0)=b=G(0)$ together with (4.6) implies

$$
\int_{-\infty}^{\infty} \int_{-\infty}^{\infty} N(1, w, F)|w|^{p-2} d u d v=\int_{-\infty}^{\infty} \int_{-\infty}^{\infty} N(1, w, G)|w|^{p-2} d u d v
$$

for some $p \in(0,1)$, since $G \in H^{p}(U)$. We use (4.24) to deduce next that

$$
F(U) \subset G(U) .
$$

Using (4.19) and (4.21), we see that

$$
K(u+i v)=\int_{-v}^{v} N(1, u+i s, G) d s-\int_{-v}^{v} N(1, u+i s, F) d s \geqq 0
$$


in the upper half plane. Fix $u \neq 0$, and put

$$
m(v)=-|u+i v|^{p-2} .
$$

Then an integration by parts gives

$$
\int_{0}^{\infty} K(u+i v) m^{\prime}(v) d v=\int_{0}^{\infty} k(u+i v)|u+i v|^{p-2} d v
$$

where

$$
k(w)=\partial K / \partial v=2 N(1, w, G)-N(1, w, F)-N(1, \bar{w}, F) .
$$

Using (4.24), we obtain

$$
\int_{-\infty}^{\infty} \int_{0}^{\infty} K(w) m^{\prime}(v) d v d u=\int_{-\infty}^{\infty} \int_{0}^{\infty} k(w)|w|^{p-2} d v d u=0 .
$$

Since $m^{\prime}>0$ and $K \geqq 0, K(w)=0$ a.e. in $\{v>0\}$.

Thus, by (4.19), (4.21) and (4.17),

$$
\int_{-v}^{v} N(1, u+i s, F) d s=\int_{-v}^{v} q(u+i s) d s=q^{*}(w)=N^{*}(1, w, G)
$$

a.e. in the upper half plane. The second term here is subharmonic in $\{v>0, u \neq b\}$ and continuous in $\{v \geqq 0\}$, as we have already noted. Since also

$$
N^{*}(1, w, G) \text { is continuous in }\{v \geqq 0\} \text {, }
$$

we must have

$$
q^{*}(w)=\int_{-v}^{v} q(u+i s) d s
$$

throughout $\{v \geqq 0\}$, and so by a simple argument (cf. last paragraph of the proof of Lemma 1.1)

$$
q(\bar{w})=q(w) \quad(w \in \boldsymbol{C}) .
$$

Using (4.18), we deduce

$$
N(1, w, F) \leqq q(w)=N(1, w, G) \quad(w \in C) .
$$

This relation implies (4.25), and we have completed the first step in the proof.

We can now define $\omega=G^{-1} \circ F$. Then $\omega(0)=0$ and we claim that $\omega: U \rightarrow U$ is an inner function $[10$, p. 24]. For $\zeta \in U$, define

$$
\omega_{\zeta}(z)=\frac{\zeta-\omega(z)}{1-\bar{\zeta} \omega(z)} .
$$

Since $\omega_{\zeta}(z)$ is subordinate to $(\zeta-z) /(1-\bar{\zeta} z)$, Jensen's theorem implies, if $\zeta \neq 0$ and $w=G(\zeta)$,

$$
N(r, w, F)=N(r, \zeta, \omega) \leqq \log \left|\frac{r}{\zeta}\right|=N(r, w, G) \quad(0<r \leqq 1) .
$$


By (4.17) and (4.34), there is equality in (4.36) when $r=1$ if $\zeta \notin \mathscr{E}$, where cap $\mathscr{E}=0$ Thus $\mathscr{E}_{0}=G^{-1}(\mathscr{E})$ also has capacity zero, and

$$
N(1, \zeta, \omega)=-\log |\zeta| \quad\left(\zeta \in U-\mathscr{E}_{0}\right) .
$$

Applying Jensen's theorem again, we deduce

$$
\lim _{r \rightarrow 1} \frac{1}{2 \pi} \int_{0}^{2 \pi} \log \left|\omega_{\zeta}\left(r e^{i \theta}\right)\right| d \theta=\lim _{r \rightarrow 1} N(r, \zeta, \omega)+\log |\zeta|=0 \quad\left(\zeta \in U-E_{0}\right) .
$$

Thus, for any such $\zeta, \omega_{\zeta}$ is a Blaschke product [14, p. 107] [7, p. 32], and it follows that $\omega$ is inner.

We conclude that

$$
F=G_{b} \circ \omega=\frac{1}{2}(1+b) \frac{1+\omega}{1-\omega}-\frac{1}{2}(1-b) \frac{1-\omega}{1+\omega}=F_{+}-F_{-}
$$

where

$$
F_{ \pm}=G_{ \pm} \circ \omega, \quad G_{ \pm}(z)=\frac{1}{2}(1 \pm b) \frac{1 \pm z}{1 \mp z}
$$

Since $G_{ \pm}$, and so also $F_{ \pm}$, map $U$ into $\{\operatorname{Re} w>0\}$, the Herglotz representations

$$
F_{ \pm}(z)=\int_{T} \frac{e^{i \varphi}+z}{e^{i \varphi}-z} d \mu_{ \pm}\left(e^{i \varphi}\right)
$$

obtain; here $\mu_{+}$and $\mu_{-}$are positive Borel measures with

$$
\mu_{ \pm}(T)=F_{ \pm}(0)=\frac{1}{2}(1 \pm b)
$$

[10, p. 3]. Recall that $F=F_{\mu}$ has the form (11); by uniqueness of $\mu\left[10\right.$, p. 4], $\mu=\mu_{+}-$ $\mu_{-}$. Further, since $\omega$ is inner,

$$
\lim _{r \rightarrow 1} \operatorname{Re} F_{+}\left(r e^{i \theta}\right)=\frac{1}{2}(1+b) \frac{1-\left|\omega\left(e^{i \theta}\right)\right|^{2}}{\left|1-\omega\left(e^{i \theta}\right)\right|^{2}}=0 \quad \text { a.e. }
$$

and thus $\mu_{+}^{\prime}\left(e^{i \theta}\right)=0$ a.e. [10, p. 4]. Similarly, $\mu_{-}^{\prime}\left(e^{i \theta}\right)=0$ a.e., so that $\mu_{+}$and $\mu_{-}$ are singular with respect to Lebesgue measure. Notice that, since $\mu_{+}$and $\mu_{-}$are positive with

$$
1=\|\mu\| \leqq\left\|\mu_{+}\right\|+\left\|\mu_{-}\right\|=\frac{1}{2}(1+b)+\frac{1}{2}(1-b)=1,
$$

$\mu_{+}$and $\mu_{-}$are mutually singular. This completes the proof of the first half of Theorem 4.2.

Conversely, given $b \in[0,1]$ and an inner function $\omega$ on $U$ with $\omega(0)=0$, we can define $F_{+}$and $F_{-}$by (4.39). Then, as above, there exist positive measures $\mu_{+}$ and $\mu_{-}$such that (4.40)-(4.42) hold. Thus $\mu_{+}$and $\mu_{-}$are singular with respect to Lebesgue measure and, if we put $\mu=\mu_{+}-\mu_{-}$, then

$$
F_{\mu}=F_{+}-F_{-}=G_{b} \subset \omega .
$$


Since $\omega$ is inner in $U, \omega_{\zeta}$ defined by (4.35) is a Blaschke product for all $\zeta \in U-\mathscr{E}_{0}$ where cap $\mathscr{E}_{0}=0$ [14, p. 111], [7, Theorem 2.15]. Since $\omega(0)=0,(4.38)$ and thus also (4.37) hold for such $\zeta[14$, p. 107], [7, p. 32]. Consequently,

$$
N\left(1, w, F_{\mu}\right)=N\left(1, w, G_{b}\right) \quad(w \in C-\mathscr{E})
$$

where cap $\mathscr{E}=0$. Now (4.6) follows from (4.14), for all $p \in(0,1)$, and we deduce $\|\mu\|=1$; thus $\|\mu\|=\left\|\mu_{+}\right\|+\left\|\mu_{-}\right\|$, so that $\mu_{+}$and $\mu_{-}$are mutually singular. The proof of Theorem 4.2 is now complete.

It might be interesting to characterize directly the measures $\mu_{+}, \mu_{-}$for which the converse part of Theorem 4.2 holds, i.e. the $\mu=\mu_{+}-\mu_{-} \in M(T)$ for which there exists some inner function $\omega$ on $U$ satisfying $F_{\mu}=G_{b} \circ \omega$ and $\omega(0)=0$. As a simple example we observe that, for $b=0$ and any $n>1$, the measures

$$
\mu_{+}=\frac{1}{2 n} \sum_{k=0}^{n-1} \delta_{\zeta_{2 k}}, \quad \mu_{-}=\frac{1}{2 n} \sum_{k=0}^{n-1} \delta_{\zeta_{2 k+1}} \quad\left(\zeta_{j}=e^{i j \pi / n}\right)
$$

correspond to $\omega(z)=z^{n}$.

Remark 1. Once (4.34) has been proved, our argument could have been based instead on the following elementary result: Let $G \in H^{p}(U)$ for some $p \in(0, \infty)$, $G \not$ constant, and let $\omega: U \rightarrow U$ be analytic with $\omega(0)=0$. Then $\omega$ is inner if and only if

$$
\int_{0}^{2 \pi}\left|G\left(\omega\left(e^{i \theta}\right)\right)\right|^{p} d \theta=\int_{0}^{2 \pi}\left|G\left(e^{i \theta}\right)\right|^{p} d \theta
$$

The proof is a slight variant on the argument given with (3.10).

Remark 2. The part of our argument proving $\omega$ inner can easily be modified to give a factorization result: Let $F$ be analytic in $U$, and let $G$ be univalent there with $G(0)=F(0)$. Then

$$
F=G \circ \omega
$$

for some inner function $\omega$ on $U$ with $\omega(0)=0$, if and only if

$$
N(1, w, F) \leqq N(1, w, G) \quad(w \in F(U))
$$

with equality holding for one $w \in F(U), w \neq F(0)$.

Proof of Theorem 4.4. Suppose $f(\not 0)$ satisfies (4.13) with equality for some $t>0$; replacing $f$ by $t^{-1} f$ shows that we may assume $t=1$. Thus $E=E_{1}(\tilde{f})$ satisfies

$$
|E|=2 \pi \Theta\|f\|_{1} \quad(>0) .
$$

To $f$ we associate the analytic function $F(z)$ defined by (8).

Following Baernstein's proof of (4.13) in [4], we let $\varphi(w)$ be the subharmonic function in $C$ which is $|\operatorname{Re} w|$ outside the strip

$$
S=\{w \in \boldsymbol{C}:|\operatorname{Im} w|<1\},
$$


and which is harmonic in $S$ with boundary values

with

$$
\varphi(w)=|\operatorname{Re} w| \quad(w \in \partial S),
$$

Baernstein proves that

$$
\varphi(w)=O(|\operatorname{Re} w|) \quad(w \rightarrow \infty) .
$$

$$
\begin{gathered}
\Theta=\varphi(0)^{-1}, \\
\varphi(F(0)) \leqq\|f\|_{1}+\varphi(0)\{1-|E| / 2 \pi\}
\end{gathered}
$$

[4, Section 2].

Using (4.44) in (4.45), we deduce $\varphi(F(0)) \leqq \varphi(0)$ and thus $F(0)=0$, since $\varphi$ is even and strictly increasing on $(0, \infty)$ [4, Section 2].

From formula (6) in [4], the boundary values of $F$ satisfy

$$
\varphi\left(F\left(e^{i \theta}\right)\right) \leqq\left|f\left(e^{i \theta}\right)\right|+\varphi(0) \chi\left(e^{i \theta}\right) \quad \text { a.e. },
$$

where $\chi$ is the characteristic function of $T-E$. Thus, using (4.44) again,

$$
\Theta \int_{0}^{2 \pi} \varphi\left(F\left(e^{i \theta}\right)\right) d \theta \leqq 2 \pi \Theta\|f\|_{1}+2 \pi-|E|=2 \pi .
$$

Since $\varphi \circ F$ is subharmonic in $U$, we deduce

$$
\Theta^{-1}=\varphi(F(0)) \leqq \frac{1}{2 \pi} \int_{0}^{2 \pi} \varphi\left(\left(F e^{i \theta}\right)\right) d \theta \leqq \Theta^{-1} .
$$

We conclude that $\varphi \circ F$ is harmonic in $U$, and this is possible only if $F(U) \subset S$. Thus $E=\left\{e^{i \theta}:\left|\operatorname{Im} F\left(e^{i \theta}\right)\right|=1\right\}$. Since $G$ defined in (4.13) is univalent in $U$ with $G(U)=S$, we can define $\omega=G^{-1} \circ F$. Then

$$
\omega: U \rightarrow U, \omega(0)=0,
$$

and it remains to prove that $\omega$ is inner, i.e. that $|E|=2 \pi$.

To see this, we start with inequality (5) in [4], which we write in the form

$$
\varphi(w)=|\operatorname{Re} w|+\varphi(0)-p(w)
$$

where $p$ is continuous in $C$ and

$$
p(w)>0 \quad(w \in S-\{0\}), \quad p(w)=\varphi(0) \quad(w \in \partial S) .
$$

Putting $w=F(z)$ and letting $|z| \rightarrow 1$, we obtain

Thus (4.44) implies

$$
\varphi\left(F\left(e^{i \theta}\right)\right)=\left|f\left(e^{i \theta}\right)\right|+\varphi(0)-p\left(F\left(e^{i \theta}\right)\right) \text { a.e. }
$$

so that

$$
\varphi(0)=\left\|\varphi\left(F\left(e^{i \theta}\right)\right)\right\|_{1}=\varphi(0)|E| / 2 \pi+\varphi(0)-\left\|p\left(F\left(e^{i \theta}\right)\right)\right\|_{1}
$$

$$
\int_{0}^{2 \pi} p\left(F\left(e^{i \theta}\right)\right) d \theta=\varphi(0)|E| .
$$


Since $p(w)=\varphi(0)$ on $\partial S$, we must have

$$
\int_{T-E} p\left(F\left(e^{i \theta}\right)\right) d \theta=0 .
$$

But $p(w)>0$ on $\bar{S}-\{0\}$, so that $|T-E|=0$, as desired.

Conversely, if $F=G \circ \omega$ where $\omega$ is an inner function on $U$, it is clear that $\left|\tilde{f}\left(e^{i \theta}\right)\right|=1$ a.e., that $|E|=2 \pi$ and that

$$
\varphi\left(F\left(e^{i \theta}\right)\right)=\left|f\left(e^{i \theta}\right)\right| \text { a.e. }
$$

Integrating, we see that $\varphi(0)=\|f\|_{1}$ if $\omega(0)=0$, and the proof of Theorem 4.4 is complete.

\section{Proofs of Theorems 4.1 and 4.3}

Suppose first that $F_{\mu}$ satisfies (4.1) and that equality holds in (4.2a) for some $r \in(0,1)$ and some $p \in(0,2)$. Using (4.14) for this choice of $r$ yields (4.24) with $N(1, w, \cdot)$ replaced by $N(r, w, \cdot)$, and the argument for (4.34) now yields

$$
N(r, w, F)=N(r, w, G) \quad(w \in \boldsymbol{C}) .
$$

Here, as in Section 4 , we have put $F=F_{\mu}, G=G_{b}=F_{v_{b}}$.

Thus

and we can put

$$
F(\{|z| \leqq r\})=G(\{|z| \leqq r\}),
$$

$$
\omega(z)=G^{-1} \circ F(z) \quad(|z| \leqq r) .
$$

Then $|\omega(z)| \leqq|z|$ in $|z| \leqq r$, and a slight variant of the argument used for (3.10), now with $\Phi(x)=\exp (p x)$, shows that equality in (4.2a) implies $|\omega(z)|=r$ on $|z|=r$. Thus, an application of the reflection principle yields that $\omega(z)$ is meromorphic in the extended plane, with

$$
\omega(z)=e^{i \beta} z B(z), \quad B(z)=\prod \frac{r\left(a_{v}-z\right)}{r^{2}-\bar{a}_{v} z} \quad\left(0 \leqq\left|a_{v}\right|<r, \beta \in \boldsymbol{R}\right) .
$$

Here the product $B(z)$ is either finite, or empty $(B(z) \equiv 1)$. In the former case, $|\omega(z)|>|z|$ in $|z|>r$, so that $M(\varrho,|\operatorname{Re} F|)=\max _{\theta}\left|\operatorname{Re} F\left(\varrho e^{i \theta}\right)\right|$ would satisfy

$$
M(\varrho,|\operatorname{Re} F|)=M(\varrho,|\operatorname{Re} G \circ \omega|)>M(\varrho,|\operatorname{Re} G|)
$$

for all $\varrho, r<\varrho<\sup \{\varrho \leqq 1: \omega(\varrho U) \subset U\}$. But Baernstein's inequality (4.2c) obviously implies

$$
M(\varrho,|\operatorname{Re} F|) \leqq M(\varrho,|\operatorname{Re} G|) \quad(0<\varrho<1),
$$

thus $B(z) \equiv 1$ in (5.2).

This completes the proof of the part of Theorem 4.1 dealing with inequality (4.2a), $0<p<2$. If $p=2$, we use the consequence of (4.21):

$$
\int_{-v}^{v} N(r, u+i s, F) d s \leqq \int_{-v}^{v} N(r, u+i s, G) d s \quad(0<v<\infty),
$$


together with (the analogue for $|z|=r$ of) formula (4.24), to deduce

$$
\int_{-\infty}^{\infty} N(r, u+i v, F) d v=\int_{-\infty}^{\infty} N(r, u+i v, G) d v \quad(u \in \boldsymbol{R}) .
$$

We now apply Baernstein's formula

$$
\int_{0}^{2 \pi}\left[\operatorname{Re} F\left(r e^{i \theta}\right)-u\right]^{+} d \theta=\int_{-\infty}^{\infty} N(r, u+i v, F) d v+2 \pi[\operatorname{Re} F(0)-u]^{+}
$$

(see [4], Section 5) with (5.4) to obtain

$$
\int_{0}^{2 \pi}\left[\operatorname{Re} F\left(r e^{i \theta}\right)-u\right]^{+} d \theta=\int_{0}^{2 \pi}\left[\operatorname{Re} G\left(r e^{i \theta}\right)-u\right]^{+} d \theta \quad(u \in \boldsymbol{R}) .
$$

But if we put $f=\operatorname{Re} F, g=\operatorname{Re} G$ and

$$
f^{*}\left(\varrho e^{i \theta}\right)==\sup _{|E|=2 \theta} \int_{E} f\left(\varrho e^{i \varphi}\right) d \varphi \quad(0<\varrho<1),
$$

then (5.6) is equivalent (cf. [2], Proposition 3) to

$$
f^{*}\left(r e^{i \theta}\right)=g^{*}\left(r e^{i \theta}\right) \quad(0 \leqq \theta \leqq \pi) .
$$

By Baernstein's arguments in Sections 5 and 6 of [4], $f^{*}$ is subharmonic in $U^{+}=$ $U \cap\{\operatorname{Im} z>0\}, g^{*}$ is harmonic there with

$$
g^{*}\left(\varrho e^{i \theta}\right)=\int_{-\theta}^{\theta} g\left(\varrho e^{i \varphi}\right) d \varphi \quad(0 \leqq \theta \leqq \pi),
$$

and $f^{*} \leqq g^{*}$ in $U^{+}$. By (5.8), we can apply Lemma 1.1 to obtain $f\left(e^{-i \alpha} z\right)=g(z)$ for some real $\alpha$; this implies (4.3) and (4.4).

Next suppose equality holds in $(4.2 b)$ for some $r \in(0,1)$ and some $p \in(1,2)$. Now the analysis depends on another formula of Baernstein ([4], Section 5):

$$
\int_{0}^{2 \pi}\left|\operatorname{Im} F\left(r e^{i \theta}\right)\right|^{p} d \theta=p(p-1) \int_{-\infty}^{\infty} \int_{-\infty}^{\infty} N(r, w, F)|v|^{p-2} d u d v+2 \pi|\operatorname{Im} F(0)|^{p} \quad(p>1),
$$

and only trivial changes in the argument already given for (4.2a), $p<2$, are required to prove Theorem 4.1 in this case.

Further, if equality holds in (4.2b) for some $r<1$ and $p=2$, precisely the argument given above for (4.2a), $p=2$, yields Theorem 4.1 in this case as well.

If equality holds in (4.2b) with $p=1$, we use

$$
\int_{-\infty}^{\infty} N(r, u, F) d u=\int_{-\infty}^{\infty} N(r, u, G) d u
$$


a simple consequence of the case $u=0$ of (5.5) with $F$ replaced by $-i F$. By (5.3), $N(r, u, F) \leqq N(r, u, G)$, so that (5.10) implies

$$
N(r, u, F)=N(r, u, G) \quad(u \in \boldsymbol{R}) .
$$

Since (4.21) holds Lemmas 4.1 and 4.2 can be applied with $p(w)=N(r, w, F)$, and we again obtain (5.1). Thus the argument given after (5.1) yields also the assertion of Theorem 4.1 for $(4.2 b), p=1$.

Next we assume equality in (4.2c) for some $r<1$ and $p>1$. There is a formula equivalent to (5.9) in which $\operatorname{Im} F$ is replaced by $\operatorname{Re} F$ and $v$ by $u$. Using this with (5.3) yields (5.4), and the argument given after (5.4) again establishes (4.3) and (4.4).

Finally, let us suppose that (4.5) holds for some $r \in\left(r_{b}, 1\right)$. Then, if we define $f, g, f^{*}, g^{*}$ as in (5.7) and (5.8), we have

$$
\int_{0}^{2 \pi} f^{+}\left(r e^{i \theta}\right) d \theta=\frac{1}{2} \int_{0}^{2 \pi}\left[\left|f\left(r e^{i \theta}\right)\right|+f\left(r e^{i \theta}\right)\right] d \theta=\int_{0}^{2 \pi} g^{+}\left(r e^{i \theta}\right) d \theta .
$$

Since $G\left(-r_{b}\right)=0, G(-r)<0<G(r)$; thus, by (4.5), $f\left(r e^{i \theta}\right)=\operatorname{Re} F\left(r e^{i \theta}\right)$ also changes sign on $0 \leqq \theta \leqq 2 \pi$, and there exists $\gamma \in(0, \pi)$ such that

$$
f^{*}\left(r e^{i \gamma}\right)=\int_{0}^{2 \pi} f^{+}\left(r e^{i \theta}\right) d \theta .
$$

By the remarks after (5.8), $f^{*} \leqq g^{*}$ in $U^{+}$so that (5.12) and (5.13) imply

$$
f^{*}\left(r e^{i \gamma}\right) \leqq g^{*}\left(r e^{i \gamma}\right) \leqq \int_{0}^{2 \pi} g^{+}\left(r e^{i \theta}\right) d \theta=f^{*}\left(r e^{i \gamma}\right) .
$$

Another appeal to the remarks after (5.8) yields $f^{*}=g^{*}$ throughout $U^{+}$, and Lemma 1.1 again implies (4.3) and (4.4).

It remains to prove Theorem 4.3; but since the functions $H_{\beta, b}$ have all the symmetry, univalence, ... properties of $G_{b}$ used in the proof of Theorem 4.1, precisely the arguments already used suffice to give Theorem 4.3 as well.

\section{Conjugate functions and rearrangements}

Let $f \in L^{1}(T)$ be real valued, and define $F$ and $\tilde{f}$ as in (8) and (9). Let $g$ be the symmetric decreasing rearrangement of $f$ :

$$
g\left(e^{i \theta}\right)=g\left(e^{-i \theta}\right)=\inf \left\{t:\left|E_{t}(f)\right| \leqq 2 \theta\right\} \quad(0 \leqq \theta \leqq \pi),
$$

where $E_{t}$ is defined in (4.12). With $g$ we associate as in (8) and (9) an analytic function $G$ and conjugate function $\tilde{g}$. In [4], Baernstein proves the following relations:

$$
\begin{gathered}
\int_{0}^{2 \pi}\left|F\left(r e^{i \theta}\right)\right|^{p} d \theta \leqq \int_{0}^{2 \pi}\left|G\left(r e^{i \theta}\right)\right|^{p} d \theta \quad(0<p \leqq 2), \\
\int_{0}^{2 \pi}\left|\operatorname{Im} F\left(r e^{i \theta}\right)\right|^{p} d \theta \leqq \int_{0}^{2 \pi}\left|\operatorname{Im} G\left(r e^{i \theta}\right)\right|^{p} d \theta \quad(1 \leqq p \leqq 2), \\
\int_{0}^{2 \pi}\left|\operatorname{Re} F\left(r e^{i \theta}\right)\right|^{p} d \theta \leqq \int_{0}^{2 \pi}\left|\operatorname{Re} G\left(r e^{i \theta}\right)\right|^{p} d \theta \quad(1 \leqq p<\infty) .
\end{gathered}
$$


In particular, if $G \in H^{p}$ for some $p \in(0,2]$, then also $F \in H^{p}$ and

$$
\begin{array}{ll}
\int_{0}^{2 \pi}\left|F\left(e^{i \theta}\right)\right|^{p} d \theta \leqq \int_{0}^{2 \pi}\left|G\left(e^{i \theta}\right)\right|^{p} d \theta & (0<p \leqq 2), \\
\int_{0}^{2 \pi}\left|\tilde{f}\left(e^{i \theta}\right)\right|^{p} d \theta \leqq \int_{0}^{2 \pi}\left|\tilde{g}\left(e^{i \theta}\right)\right|^{p} d \theta & (1 \leqq p \leqq 2) .
\end{array}
$$

It is easy to see that Baernstein's methods in [4, Section 6] also yield the inequalities

$$
\begin{aligned}
& \int_{0}^{2 \pi}\left|F\left(e^{i \theta}\right)\right|^{p} d \theta \geqq \int_{0}^{2 \pi}\left|G\left(e^{i \theta}\right)\right|^{p} d \theta \quad(p>2) \\
& \int_{0}^{2 \pi}\left|\tilde{f}\left(e^{i \theta}\right)\right|^{p} d \theta \geqq \int_{0}^{2 \pi}\left|\tilde{g}\left(e^{i \theta}\right)\right|^{p} d \theta \quad(p>2),
\end{aligned}
$$

provided $f \in L^{p}(T)$. A proof of (6.3) is given below, in the proof of Theorem 6.2. Simple examples (e.g. $f=\cos 2 \theta$ ) show, however, that the analogue for $r<1$ of (6.3) cannot be true.

Theorem 6.1. The inequalities (6.1) are all strict, for each $r \in(0,1)$ and admissible $p$, unless

$$
f\left(e^{i \theta}\right)=g\left(e^{i(\theta+\alpha)}\right) \text { a.e. }
$$

for some real $\alpha$.

As in the statements of Theorems 4.1 and 4.3, we do not regard as admissible the choice $p=1$ in (6.1c), unless $r$ is such that $\operatorname{Re} F\left(r e^{i \theta}\right)$ or $\operatorname{Re} G\left(r e^{i \theta}\right)$ changes sign on $0 \leqq \theta \leqq 2 \pi$. The proof of Theorem 6.1 is the same as (the first part of) that of Theorem 4.1, and we omit it.

Theorem 6.2. Assume that equality holds in one of the inequalities in (6.2) and (6.3) for some admissible $p, p \neq 2$, for which the integrals involved are finite. Then

$$
f\left(e^{i \theta}\right)=g\left(\omega\left(e^{i \theta}\right)\right) \text { a.e. }
$$

for some inner function $\omega$ on $U$ with $\omega(0)=0$.

Conversely, if $f$ has the form (6.5) for some such $\omega$, then equality holds throughout (6.2) and (6.3).

We observe the following

Corollary. Let $E \subset T$ be measurable and put $E^{*}=\left\{e^{i \theta}:|\theta|<(1 / 2)|E|\right\}$. Then there exists an inner function $\omega$ on $U$ such that, for almost all $\theta$,

$$
\omega\left(e^{i \theta}\right) \in E^{*} \Leftrightarrow e^{i \theta} \in E .
$$


For, if we choose $f=\chi_{E}=$ characteristic function of $E$, then $g=\chi_{E^{*}}$ and, by a well known result of Stein and Weiss [22], $\tilde{g}$ and $\tilde{f}$ have the same distribution function. Thus there is equality in (6.2b) and (6.3b) for all $p>0$. Now Theorem 6.2 implies

$$
\chi_{E}\left(e^{i \theta}\right)=\chi_{E^{*}}\left(\omega\left(e^{i \theta}\right)\right) \quad \text { a.e. }
$$

for some inner function $\omega$ on $U$, proving (6.6).

Baernstein has shown us (personal communication) a function-theoretic proof of the Stein-Weiss theorem which gives an alternate proof of the Corollary. See also [6] for a related result.

Proof of Theorem 6.2. We first observe that the key formulas and inequalities used in Sections 4 and 5 remain valid for the present choices of $F$ and $G$ (cf. [4], Section 6). In particular, the fact that $f$ and $g$ are equidistributed shows that (5.6) holds with $r=1$; using (5.5) with $r=1$ then yields (5.4) with $r=1$. Thus if we put

then (5.3) implies

$$
K_{1}(w)=\int_{v}^{\infty} k(u+i s) d s \quad(w=u+i v, v>0),
$$

$$
K_{1}(w)=-K(w) \leqq 0 \quad(w=u+i v, v>0)
$$

where $K$ and $k$ are defined as in (4.26), (4.28).

Assume equality holds in $(6.2 \mathrm{~b})$ or $(6.3 \mathrm{~b})$ for some $p \in(1, \infty), p \neq 2$. Letting $r \rightarrow 1$ in formula (5.9) then yields

$$
\int_{-\infty}^{\infty} \int_{-\infty}^{\infty} N(1, w, F)|v|^{p-2} d u d v=\int_{-\infty}^{\infty} \int_{-\infty}^{\infty} N(1, w, G)|v|^{p-2} d u d v
$$

where the integrals are finite.

If $1<p<2$, the integration by parts argument given in (4.27) remains valid here, now with $m(v)=-v^{p-2}$, and we deduce $K(w)=0$ a.e. in $\{\operatorname{Im} w>0\}$.

When $p>2$, the same argument applied to $K_{1}(w)$ yields

$$
\begin{gathered}
\int_{0}^{\infty} K_{1}(u+i v) d\left(v^{p-2}\right)=\int_{0}^{\infty} v^{p-2} k(u+i v) d v, \\
(p-2) \int_{-\infty}^{\infty} \int_{0}^{\infty} v^{p-3} K_{1}(u+i v) d v d u=\int_{-\infty}^{\infty} \int_{0}^{\infty} v^{p-2} k(u+i v) d v d u,
\end{gathered}
$$

and (6.7) and (6.8) together with (5.9) again imply $K(w)=0$ a.e.

From the definition of $K$ we see that (4.30) remains valid here, with $q$ again being given by (4.16), and we deduce (4.34) and its consequence (4.25). Now the arguments in (4.35)-(4.38) yield

$$
F(z)=G(\omega(z)) \quad(|z|<1)
$$

where $\omega: U \rightarrow U$ is an inner function with $\omega(0)=0$. 
Since $G$ is univalent, Lindelöf's Theorem [7, pp. 19, 56] shows that

$$
f\left(e^{i \theta}\right)+i \tilde{f}\left(e^{i \theta}\right)=\lim _{r \rightarrow 1} G\left(\omega\left(r e^{i \theta}\right)\right)=G\left(\lim _{r \rightarrow 1} \omega\left(r e^{i \theta}\right)\right)
$$

a.e., and the assertion of Theorem 6.2 for $(6.2 b)-(6.3 b)$ and $p \neq 1$ is proved.

The same arguments, using (4.24) instead of (6.8), yield the assertion of the theorem for $(6.2 \mathrm{a})-(6.3 \mathrm{a})$ and $0<p<\infty, p \neq 2$.

Remark. To prove inequality (6.3b), observe first that, for $p>2$, (6.7) and (6.9) imply (6.8) with $\geqq$ instead of $=$. (All integrals considered are finite, since we have assumed $f \in L^{p}$.) Then (5.9) and the definition (4.28) of $k(w)$ imply (6.3b). The proof of (6.3a) is similar.

It remains to consider the case of equality in (6.2b), $p=1$. We use (5.10) with $r=1$ to deduce (5.11), a.e. in $-\infty<u<\infty$. Since $G$ is univalent, the argument given after (5.11) shows that, outside a set of capacity zero, we have

$$
q(w)=N(1, w, F)=N(1, w, G)
$$

(cf. (4.17)). Thus (4.25) holds, and we can again deduce (6.10) and (6.11) as above.

The converse assertion in Theorem 6.2 is proved by the argument in the last paragraph of the proof of Theorem 4.2.

\section{References}

[1] Baernstein, A.: A generalization of the $\cos \pi \varrho$-theorem. - Trans. Amer. Math. Soc. 193, 1974, $181-197$.

[2] Baernstein, A.: Integral means, univalent functions and circular symmetrization. - Acta Math. 133, 1974, 133-169.

[3] Baernstein, A.: Regularity theorems associated with the spread conjecture. - J. Analyse Math. XXXI, 1977, 76-111.

[4] Baernstein, A.: Some sharp inequalities for conjugate functions. - Indiana Univ. Math. J. 27, 1978, 833-852.

[5] Baernstein, A., and B. A. TAYlor: Spherical rearrangements, subharmonic functions, and ${ }^{*}$-functions in $n$-space. - Duke Math. J. 43, 1976, 245-268.

[6] Cereteli, O. D.: Certain properties of inner functions. - Sakharth. SSR Mecn. Akad. Moambe 82,1976 , no. $2,313-316$.

[7] Collingwood, E. F., and A. J. Lohwater: The theory of cluster sets. - Cambridge Univ. Press, London, 1966.

[8] Davis, B.: On the weak type $(1,1)$ inequality for conjugate functions. - Proc. Amer. Math. Soc. 44, 1974, 307-311.

[9] DAvis, B.: On Kolmogorov's inequalities $\|\tilde{f}\|_{p} \leqq C_{p}\|f\|_{1}, 0<p<1$. - Trans. Amer. Math. Soc. 222, 1976, 179-199.

[10] Duren, P.: Theory of $H^{p}$ spaces. - Academic Press, New York-London, 1970.

[11] EDREI, A., and W. Fuchs: Asymptotic behavior of meromorphic functions with extremal spread I, II. - Ann. Acad. Sci. Fenn. Ser. A I 2, 1976, 67-111; 3, 1977, 141-168.

[12] Esś́n, M.: The $\cos \pi \lambda$-theorem. - Lecture Notes in Mathematics 467, Springer-Verlag, Berlin-Heidelberg-New York, 1975. 
[13] Essén, M., and D. F. SHEA: An extremal problem in function theory. - Proc. Conf. in Complex Analysis, Univ. of Kentucky, Lecture Notes in Mathematics 599, Springer-Verlag, Berlin-Heidelberg-New York, 1977.

[14] Frostman, O.: Potentiel d'équilibre et capacité des ensembles avec quelques applications à la théorie des fonctions. - Meddel. Lunds Univ. Mat. Sem. 3, 1935, 1-118.

[15] GARIEPY, R., and J. L. LeWIS: Space analogues of some theorems for subharmonic and meromorphic functions. - Ark. Mat. 13, 1975, 91-105.

[16] Hayman, W. K.: Multivalent functions. - Cambridge Univ. Press, London, 1958.

[17] Hayman, W. K.: Meromorphic functions. - Oxford Math. Monograph, Clarendon Press, Oxford, 1964.

[18] Hayman, W. K., and P. B. Kennedy: Subharmonic functions, I. - Academic Press, New York-London, 1976.

[19] Helms, L. L.: Introduction to potential theory. - Wiley-Interscience, New York-LondonSydney-Toronto, 1969.

[20] Jenkins, J. A.: Some uniqueness results in the theory of symmetrization. - Ann. of Math. $61,1955,106-115$.

[21] Lehto, O.: A majorant principle in the theory of functions. - Math. Scand. 1, 1953, 5-17.

[22] Stein, E. M., and G. Weiss: An extension of a theorem of Marcinkiewicz and some of its applications. - J. Math. Mech. 8, 1959, 263-284.

Royal Institute of Technology

Department of Mathematics

S-100 44 Stockholm 70

Sweden

Received 18 December 1978
University of Wisconsin

Department of Mathematics

Madison, Wisconsin 53706

USA 